\title{
Optimal Design and Analysis of Cross Hinge Four-Bar Mechanism Based on Spatial Switching Method*
}

\author{
Bin WANG**, Lin-Zhen ZHOU**, Cong-Dong JI***, Hong-Da HU** \\ and Xin-Bo CHEN**** \\ ** UGS School, Yancheng Institute of Technology, Yancheng 224051, PR China \\ E-mail: wangbin09@yahoo.com.cn \\ *** School of Automotive and Traffic Engineering, Jiangsu University, \\ Zhenjiang 212013, PR China \\ **** School of Automotive Studies, Tongji University, Shanghai 201804, PR China
}

\begin{abstract}
Through dynamic simulation of the cross-hinge four-bar mechanism, it is found the primary reason for unbalance of the mechanism results from the unequal swing angle of driving link and driven link. To simplify analysis and calculation, a spatial switching approach was proposed to switch nonlinear problem into linear problem, by switching the problem of unequal swing angle of driving link and driven link into the problem of length variation of connecting link. Therefore, the length of driving link, the degree of its angle of intersection, and the amplitude of swing are three important parameters that influence the dynamic balance of the mechanism. An optimal design software was designed on the basis of establishment of target function and constraint conditions. Finally, through optimal calculation of the design software and Adams simulation, a case was employed to test the efficiency of the optimal design approach.
\end{abstract}

Key words: Cross-Hinge Four Bar Mechanism, Spatial Switching Approach, Dynamic Analysis, Optimal Design

\section{Introduction}

Cross hinge four-bar mechanism is one of the main forms of plane hinge four-bar mechanism, which is commonly used in cutting processing equipment of clothing, shoes and hats. As the requirements of processing range and accuracy are increasingly rigid for cutting processing equipment, customizing processing equipment with different sizes and different accuracy requirements is often needed. Equipment manufacturing enterprises usually adopt manual mapping measurements and estimation methods for product design. As a result, there are many problems, such as low design accuracy, low design efficiency, large errors, among others. Planar four-bar mechanism has the advantages of simple structure, diverse types and wide application. Its kinematic geometry is always the base of mechanism teaching and scientific research. It is also one of the topics which the agency scholars are interested in, and it is one of the most representative planar four-bar mechanisms. Many scholars have done much research on the planar four-bar mechanism. For example, Michael E. et $\mathrm{al}^{(1)}$ proposed and analyzed that the diversity of planar four-bar

${ }^{*}$ Received 13 Jan., 2013 (No. 13-0011) [DOI: 10.1299/jamdsm.7.305]

Copyright $@ 2013$ by JSME 
mechanism leads to a number of different mechanical properties. Tokuz Dulger L.C. ${ }^{(2)}$ established the mathematical model of the motor-driven, four-bar mechanism and proposed a new method of calculation based on this model. Karkoub M., et al ${ }^{(3)}$ conducted the dynamic modeling for a flexible four-bar mechanism and studied the design of controller.

The use of mathematical optimization method and computer aided calculation for synthesis of mechanism has developed rapidly since the late fifties. There are three main aspects that reflect the whole process of optimal combination. They are the establishment of mathematical model, selection of optimization methods and computer programming. But, currently, there are no special studies that regard the above three aspects as a unified system according to the classification of institutional structure. This work is of great practical significance because it not only contributes to the application and extension of institutional optimum synthesis, but also helps to promote institutional optimum synthesis as an independent sub-discipline in the field of theoretical mechanics. In view of this, based on their thorough understanding of the characteristics of planar four-bar mechanism, Li Y.X. et $\mathrm{al}^{(4)}$ put forward a method which employs the man-machine interaction for mechanism description and realizes the automatic computer aided modeling for the linkage mechanism after the general analytic method for planar linkage mechanism is utilized. In Ref. (5), Chen X.B. and Li Y. obtained some CV (constant velocity) plane hinge linkages with a variable distance between the input and output axes through the combination of simple CV plane hinge linkages. In addition the paper presents the double crank conditions of these linkages (including general Schmidt Coupling) and proves that they have the characteristic of CV transmission. Sun X.B. et al ${ }^{(6)}$ from Huazhong University of Science and Technology, in the light of the disadvantage of the conventional four-bar mechanism having less controllable parameters, provided a new four-bar mechanism with variable parameters whose structure parameters change regularly during exercise, and deduced two important mathematic models for the analysis and design of four-bar mechanism with variable parameters. Liu A.X.and Yang T.L. ${ }^{(7)}$ proposed a new method for approximating the shaking force and shaking moment balancing of planar linkages.

The proposed cross hinge four-bar mechanism has antisymmetric particularity. Because it is difficult to use common four-bar mechanism optimization methods for the parameter optimization, they will not be used. (Such examples include the improved ant colony algorithm $\operatorname{method}^{(8)}$, neural network optimization $\operatorname{method}^{(9)}$, fast fourier transform $\operatorname{method}^{(10)}$, numerical atlas method ${ }^{(11)}$, differential evolution method ${ }^{(12)}$ and orientation cosine matrix method ${ }^{(13)}$ ). Therefore, this paper presents an optimization design method based on space transformation focusing on the optimization design of cross hinge four-bar mechanism. The optimization design method converts the nonlinear problems based on different swing angles into the linear problems, and according to the theoretical analysis, the corresponding optimization software is written, which helps to obtain the optimization design data of the cross hinge four-bar balance mechanism quickly and easily. Finally, based on the commercial soft platform ADAMS, kinematics simulation model and the corresponding man-machine operation interface for cross hinge four-bar mechanism are established by the application of the parameter-driving technology. Through the modeling and motion simulation, the precise and more efficient mapping of four-bar mechanism design is conveniently and quickly acquired. Therefore the accurate data of design is offered for four bar linkage mechanism design ${ }^{(14)}$. This result would play an instructive role in its engineering significance and has application value both in the lectotype of motion trail and the dimensional synthesis of four bar linkage mechanism.

Nomenclature 


\begin{tabular}{|c|c|}
\hline$L_{1}$ & Length of the input link $1(\mathrm{~mm})$ \\
\hline$L_{2}$ & Length of the coupler link $2(\mathrm{~mm})$ \\
\hline$L_{3}$ & Length of the output link $3(\mathrm{~mm})$ \\
\hline$L_{4}$ & Length of the ground link $4(\mathrm{~mm})$ \\
\hline$\theta_{1}$ & Angle of the balanced link 1 (counter clockwise) (deg) \\
\hline$\theta_{2}$ & Angle of the balanced link 2 (counter clockwise) (deg) \\
\hline$\theta_{3}$ & Angle of the balanced link 3 (counter clockwise) (deg) \\
\hline$\alpha$ & Angle between long and short links(deg) \\
\hline$\omega_{1}$ & Angular velocity of link 1 (deg/s) \\
\hline$\omega_{2}$ & Angular velocity of link 2 (deg/s) \\
\hline$\omega_{3}$ & Angular velocity of link 3 (deg/s) \\
\hline$\partial_{3}$ & Angular acceleration of link $3\left(\mathrm{deg} / \mathrm{s}^{2}\right)$ \\
\hline$\beta$ & Instantaneous angle rotation changes of link $1(\mathrm{deg})$ \\
\hline$\beta^{\prime}$ & Instantaneous angle rotation changes of link 3 (clockwise) (deg) \\
\hline$\beta^{\prime \prime}$ & Instantaneous angle rotation changes of link 3 (counter clockwise) (deg) \\
\hline
\end{tabular}

\section{Motion analysis of cross hinge four-bar mechanism}

In order to conduct the theoretical analysis of cross hinge four-bar mechanism, a certain type of cutting processing equipment is simplified, and the entity models of its key components are established by using NX software (as shown in Fig.1). Figure 1 displays the location of the cross hinge four-bar mechanism and the lifting platform, which facilitates the analysis and research of its principles of movement.
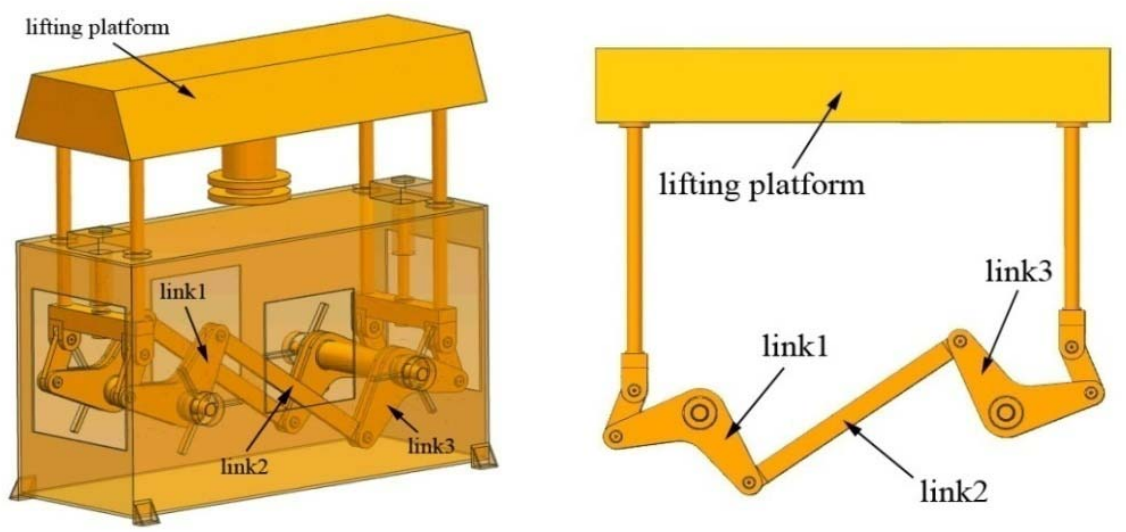

Fig.1. Simplified entity model of cutting equipment

Figure 2 is the movement schematic diagram of the lifting platform driven by the cross 
hinge four-bar mechanism. Let link 1 be the driving link. When rotating counterclockwise around the point $O$, link 1 causes the rotation of the connecting link 2, and the connecting link 2 causes the counterclockwise rotation of the link 3 around the point $O_{1}$, thus causing the upward movement of the lifting platform GH. Similarly, the lifting platform GH moves downward when link 1 rotates clockwise around the point $O$.

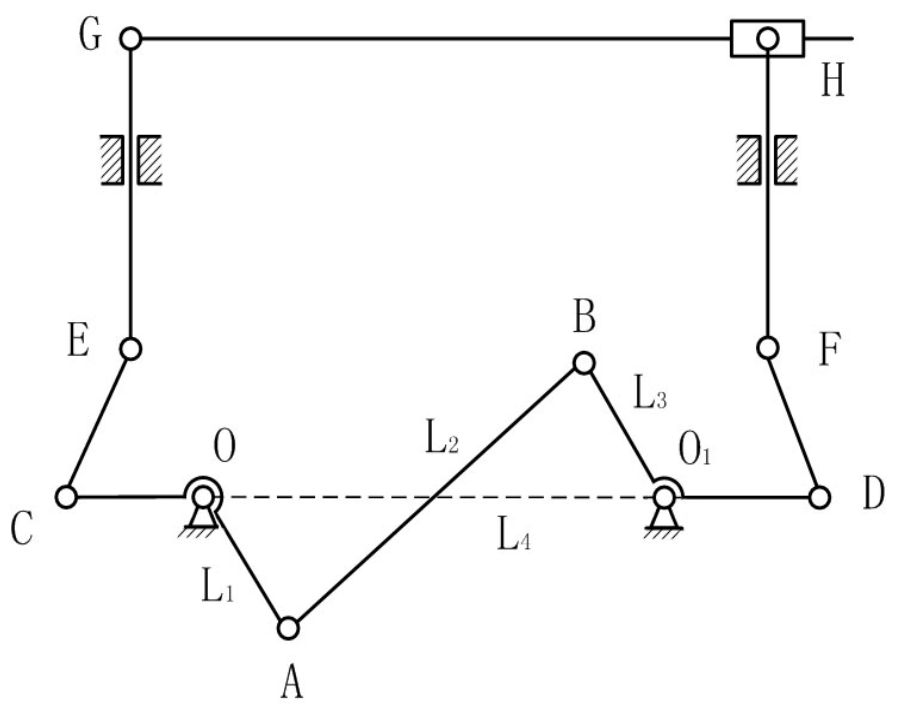

Fig. 2. The movement schematic diagram of the lifting platform driven by the cross hinge four-bar mechanism

As shown in Fig.3, the point $O$ acts as the coordinate origin, and $O O_{1}$ as the $\mathrm{x}$ axis. This establishes the rectangular coordinate system (x,y). $A_{0}$ and $B_{0}$ are the balanced static positions of the endpoints of the links 1 and 3. $\theta_{1}$ is the angle of the link 1 in its anti-symmetric state in the coordinate system, and $\theta_{2}$ and $\theta_{3}$ are the angles of the links 2 and 3 in their balanced states in the coordinate system.

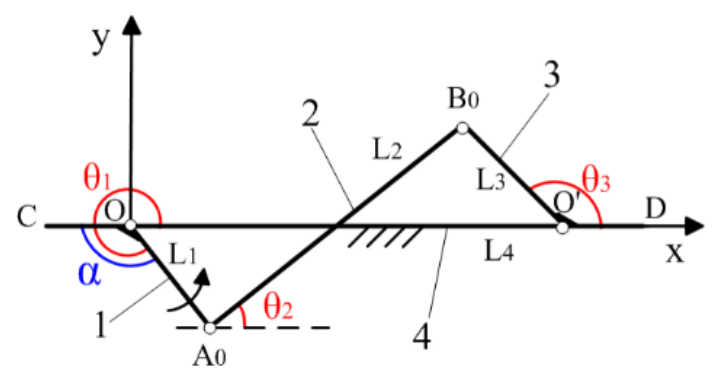

Fig.3. Cross hinge four-bar mechanism diagram

According to the geometric relationship of the cross hinge four-bar mechanism, the following formulas can be obtained:

$$
\left\{\begin{array}{l}
L_{1} \cos \theta_{1}+L_{2} \cos \theta_{2}-L_{3} \cos \theta_{3}-L_{4}=0 \\
-L_{1} \sin \theta_{1}-L_{2} \sin \theta_{2}+L_{3} \sin \theta_{3}=0
\end{array}\right.
$$

When $\theta_{1}$ is given, $\theta_{2}$ and $\theta_{3}$ can be obtained according to the above formulas.

By using the derivation of the above two formulas, and because the angular velocity $\omega_{1}$ of the link 1 is constant, the angular velocity $\omega_{2}$ of the link 2 and the angular velocity $\omega_{3}$ of the link 3 can be obtained respectively:

$$
\omega_{2}=\frac{L_{1} \sin \left(\theta_{1}-\theta_{3}\right)}{L_{2} \sin \left(\theta_{3}-\theta_{2}\right)} \omega_{1}
$$




$$
\omega_{3}=\frac{L_{1} \sin \left(\theta_{1}-\theta_{2}\right)}{L_{3} \sin \left(\theta_{3}-\theta_{2}\right)} \omega_{1}
$$

By using the derivation of $\omega_{3}$, and bringing $\omega_{2}$ into it, the angular acceleration $\partial_{3}$ of the link 3 can be obtained:

$$
\partial_{3}=\left[\frac{L_{1} \cos \left(\theta_{1}-\theta_{2}\right)}{L_{3} \sin \left(\theta_{3}-\theta_{2}\right)}-\frac{L_{1}^{2} \cos \left(\theta_{3}-\theta_{2}\right) \sin ^{2}\left(\theta_{1}-\theta_{2}\right)}{L_{3}^{2} \sin ^{3}\left(\theta_{3}-\theta_{2}\right)}-\frac{L_{1}^{2} \sin ^{2}\left(\theta_{1}-\theta_{3}\right)}{L_{2} L_{3} \sin ^{3}\left(\theta_{3}-\theta_{2}\right)}\right] \omega_{1}^{2}
$$

3. The establishment of cross hinge four-bar optimization design model and the development of software

\subsection{The determination of design variables}

The motion characteristic of cross hinge four-bar mechanism completely depends on the length ratio of the four links, because in the actual structure, the length of the center distance $L_{4}$ is often given in advance, and $L_{1}=L_{3}$. Therefore, the actual variables can be consolidated into two design variables: (1) $L_{1}$ (the length of the short link 1) and (2) $\alpha$ (the angle of long and short links), which are indicated as $X=\left[x_{1}, x_{2}\right]^{T}=\left[L_{1}, \alpha\right]^{T}$. In the balanced state, the relationship between $\alpha, \theta_{1}$ and $\theta_{3}$ can be described by following equation: $\alpha=\theta_{3}=\theta_{1}-180^{\circ}$.

\subsection{The establishment of the objective function}

Because of the connecting link 2, the moving angles of link 1 and 3 at the left and right sides of the mechanism are not always equal, and there is no linear relationship between the difference of angle changes and the unbalanced degree of the mechanism. To facilitate the analysis, we need to understand that the moving angles of link 1 and 3 are unequal and difficult to analyze and measure, so we convert this unequal angle problem into the length changing problem of the connecting link 2. Fig.4 is the former kinematic sketch of cross hinge four-bar mechanism before conversion. The method of changing the length of link 2 is used to simplify the problem, and its kinematic sketch is shown as Fig.5. Suppose that the link 2 is disconnected, and the instantaneous rotation angles of the links 1 and 3 are equal, which are expressed by $\beta$. Let $A_{0}$ and $B_{0}$ be the balanced positions of the endpoints of the links 1 and 3 in the mechanism; and let $A$ and $B$ be the dynamic positions of the endpoints of links 1 and 3 (as shown in Fig.5). $\left(x_{A}, y_{A}\right)$ and $\left(x_{B}, y_{B}\right)$ are the coordinates of their dynamic locations, and they satisfy the following equations:

$$
\left\{\begin{array}{l}
A:\left(-L_{1} \cos \left(\theta_{3}+\beta\right),-L_{1} \sin \left(\theta_{3}+\beta\right)\right) \\
B:\left(L_{4}+L_{1} \cos \left(\theta_{3}-\beta\right), L_{1} \sin \left(\theta_{3}-\beta\right)\right)
\end{array}\right.
$$

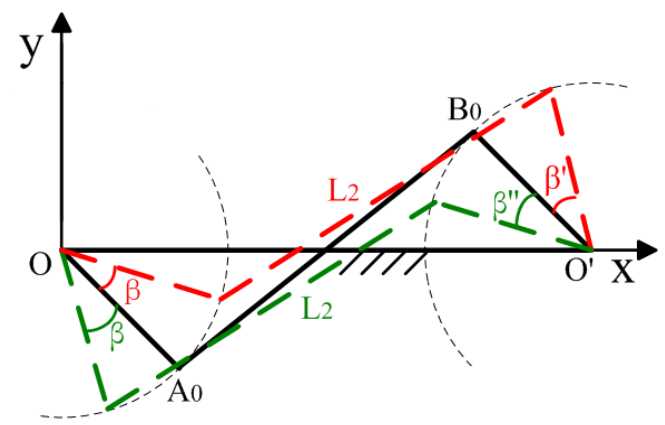

Fig.4. Former kinematic sketch of cross hinge four-bar mechanism 


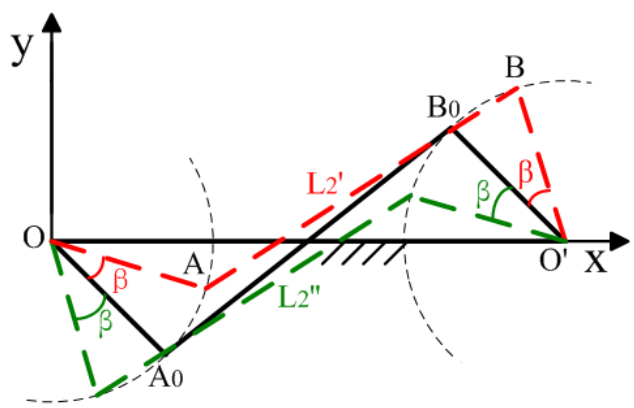

Fig.5. Kinematic sketch of cross hinge four-bar mechanism after conversion

When links 1 and 3 are in equilibrium, namely $\beta=0$, the coordinates of $A_{0}$ and $B_{0}$ can be simplified as:

$$
\left\{\begin{array}{l}
A_{0}:\left(-L_{1} \cos \theta_{3},-L_{1} \sin \theta_{3}\right) \\
B_{0}:\left(L_{4}+L_{1} \cos \theta_{3}, L_{1} \sin \theta_{3}\right)
\end{array}\right.
$$
$\overline{A_{0} B_{0}}$ is

Therefore, the variation $\delta$ of the distance $\overline{A B}$ between $A$ and $B$ relative to

$$
\delta=\left|\overline{A B}-\overline{A_{0} B_{0}}\right|
$$

Using the sum and difference of product formula, Eq.(7) can be simplified as:

$$
\begin{aligned}
& \delta=\mid \sqrt{L_{4}^{2}+4 L_{1} L_{4} \cos \theta_{3}+4 L_{1}^{2}}- \\
& \sqrt{L_{4}^{2}+4 L_{1} L_{4} \cos \theta_{3} \cos \beta+4 L_{1}^{2} \cos ^{2} \beta} \mid
\end{aligned}
$$

The optimization design take the minimum variance of the variation $\delta$ of the distance $\overline{A B}$ between $A$ and $B$ relative to $\overline{A_{0} B_{0}}$, and the established objective functions are as follows:

$$
\min f(X)=\frac{1}{n-1} \sum_{i=1}^{n} \delta_{i}^{2}
$$

\subsection{Determination of constraint conditions}

Crow hinge four-bar mechanism is a special kind of crank and rocker mechanism. Due to the small swing angle of its driving link 1, it can't be collinear with the link 4 . The constraints may not consider the existence of crank and the minimum transmission angle, but only need to consider the boundary conditions of the design variables:

$$
\min x_{i} \leq x_{i} \leq \max x_{i} \quad(i=1,2)
$$

In the above formula, $\min x_{i}$ and $\max x_{i}$ are the upper and lower limits of the $\mathrm{i}^{\prime}$ th variable.

\subsection{Design and development of optimization software}

According to the proposed new optimization theory based on $\mathrm{CH}$, the optimization system of cross hinge four-bar mechanism is developed to facilitate the numerical calculation ${ }^{(15)}$. Users only need to input center distance, length range of short link, angle swing amplitude of short link, and angle range of long and short links according to the device dimensions. This system will analyze and optimize the cross hinge four-bar mechanism automatically, and output the optimum parameter combination including the length of short link, angles between long and short links, and error accuracy. .

The calculation flow chart of the software is shown in Fig.6, where $i$ is the length of short link, $\mathrm{j}$ is the length of long link, $\mathrm{k}$ is the swinging angle of driving link 1 , and $\mathrm{d}(\mathrm{i}, \mathrm{j}, \mathrm{k})=$ $\delta, \mathrm{e}(\mathrm{i}, \mathrm{j})=f(\mathrm{X})$.

(1) On the software interface, users input the range of $\mathrm{i}, \mathrm{j}$ and $\mathrm{k}$ according to their needs, and then the program will determine whether the data given by users is reasonable or not.

(2) If the input data meets the requirements, the next step is to choose the operation 
accuracy and click the operation button to start.

(3) The data within the range given by users will be calculated and compared one by one, until the optimal combination is obtained.

(4) Save data, display the optimal combination and end the process.
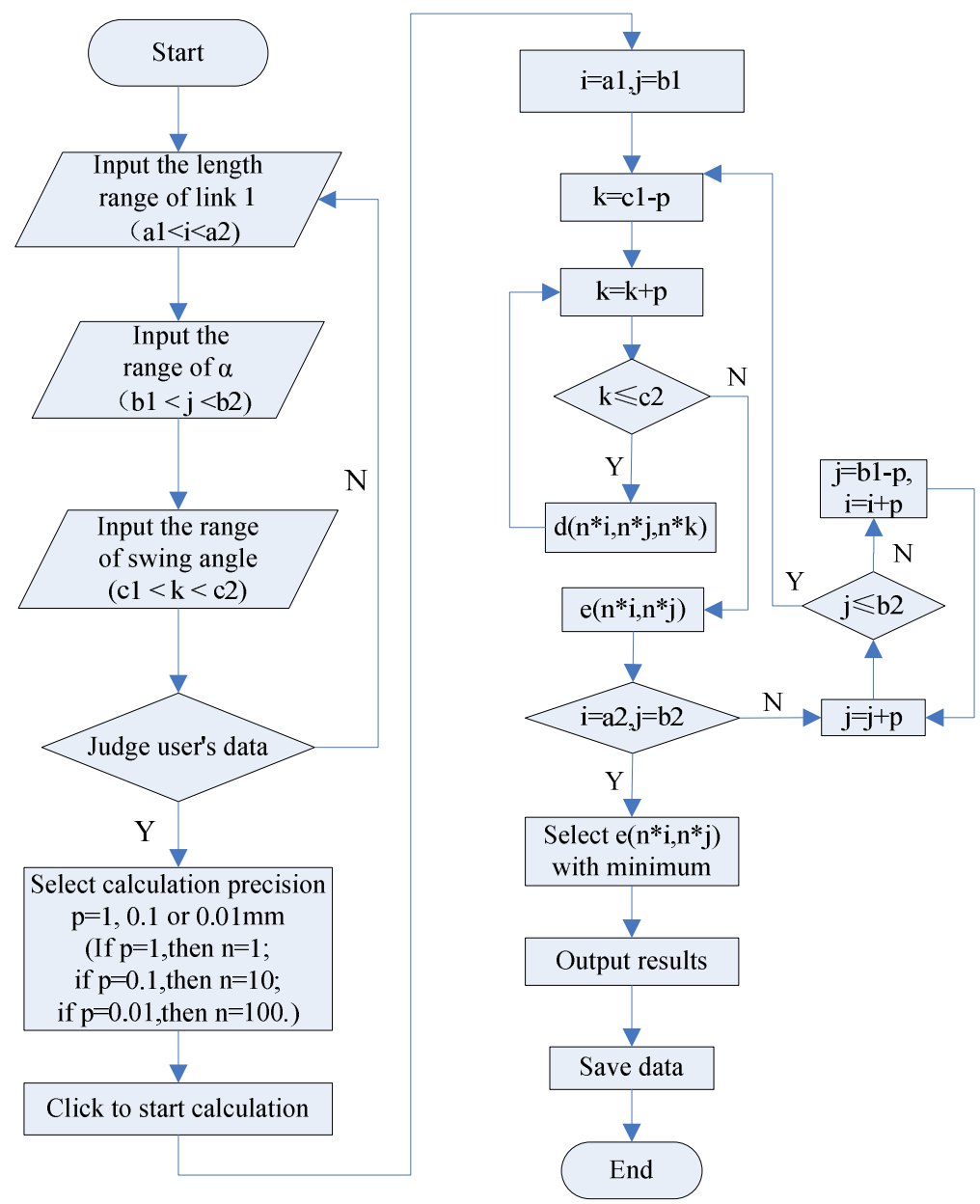

Fig.6. Calculation flow chart of software

The software has the following functions to, automatically design and rapidly display the optimum parameter combination of cross hinge four-bar balance mechanism including the length of short link, angles between the long and short links, and error accuracy, according to the center distance given by users. As a result, accuracy and efficiency requirements of user customized cutting equipment are ensured.

\section{The optimal design example of cross hinge four-bar mechanism}

Example: according to the overall design requirements of the machine, set the center distance $L_{4}=1128.53 \mathrm{~mm}$, allowing the design angle $\alpha=\theta_{1}-180^{\circ}$ of the short and long side ranges from $100^{\circ}$ to $120^{\circ}$, and allowing the length $L_{1}=L_{3}$ of the short side ranges from $200 \mathrm{~mm}$ to $220 \mathrm{~mm}$. The required swing angle of the short link is $\pm 15^{\circ}$. In the mechanism, the angle $\alpha$ of the short and long links and the length $L_{1}$ of the short link are required so that the mechanism works more smoothly. Then calculate the length of the oblique link 2. The parameters users need are provided in Table 1.

Table 1. Parameters provided by users in the example

\begin{tabular}{lcll}
\hline $\mathbf{L}_{\mathbf{1}}(\mathbf{m m})$ & $\mathbf{L}_{\mathbf{4}}(\mathbf{m m})$ & $\boldsymbol{\alpha}(\mathbf{d e g})$ & $\boldsymbol{\beta ( d e g )}$ \\
\hline $200 \sim 220$ & 1128.53 & $100 \sim 120$ & \pm 15 \\
\hline
\end{tabular}


The optimization design of this example is as follows:

(1) The formula (8) is written into the computer program. Its interface design is shown in $\operatorname{Fig.} 7^{(16)}$

(2) In choosing the most accurate iteration process, considering the computing task is time-consuming, generally the length iteration accuracy is given as $0.01 \mathrm{~mm}$, and the angle iteration accuracy is given as $0.01^{\circ}$;

(3) As shown in Fig.7, in the program interface, the center distance length $L_{4}=1128.53 \mathrm{~mm}$ is inputted, and similarly the bounds of $\alpha=\theta_{1}-180^{\circ}$ are $100^{\circ} \sim 120^{\circ}$, the bounds of $L_{1}=L_{3}$ are $200 \sim 220 \mathrm{~mm}$, and the swing angle is $\pm 15^{\circ}$;

(4) Each group of data is calculated in this program through the control variable method and the results are saved. Part of the calculation results are shown in Table 2. Then through comparative analysis, the optimal combination is obtained as follows: $L_{1}=200.01 \mathrm{~mm}$, $\alpha=110.48^{\circ}, L_{2}=1057.213197675811 \mathrm{~mm}$; the variance and average value of the dynamic variation $\delta$ of $L_{2}$ are 0.0000885690 and 0.0075814674 .

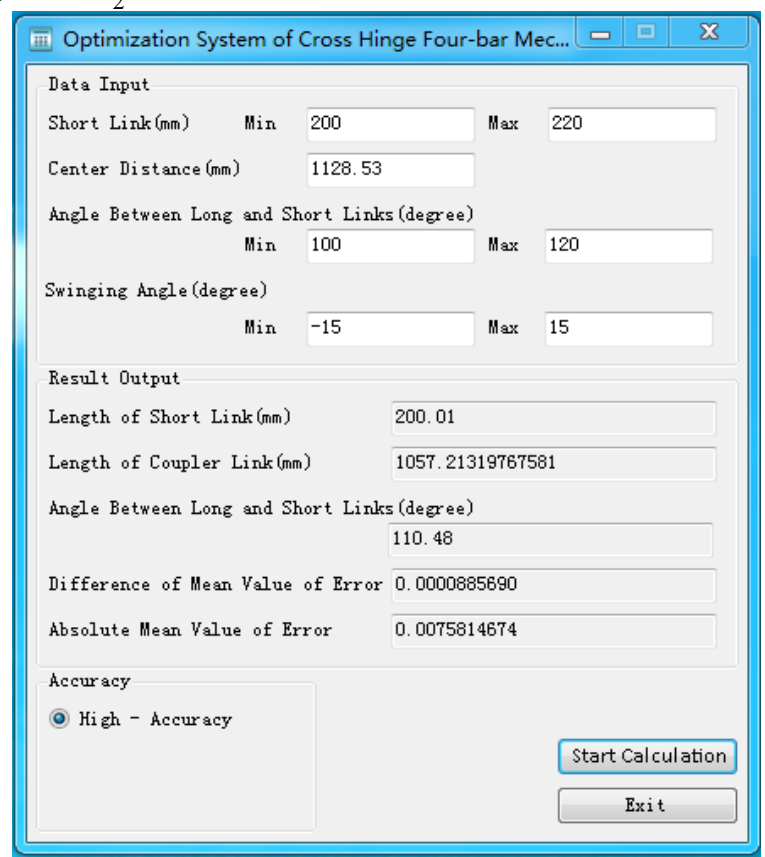

Fig.7. The optimization system interface of cross hinge four-bar mechanism

Table 2. Part of the calculation results

\begin{tabular}{cccc}
\hline $\begin{array}{c}\text { Length of Short } \\
\text { Link (mm) }\end{array}$ & $\begin{array}{c}\text { Length of Oblique } \\
\text { Link (mm) }\end{array}$ & $\begin{array}{c}\text { Angle Between } \\
\text { Long and Short } \\
\text { Links (deg) }\end{array}$ & $\begin{array}{c}\text { Difference of Mean } \\
\text { Square Errors }\end{array}$ \\
\hline 200.00 & 1129.95850301948 & 100.00 & 1.31314834256116 \\
200.00 & 1129.88983635641 & 100.01 & 1.31074543217922 \\
200.00 & 1129.82116763553 & 100.02 & 1.30834451750548 \\
200.00 & 1129.75249685857 & 100.03 & 1.30594559912898 \\
200.00 & 1129.68382402724 & 100.04 & 1.30354867763774 \\
200.00 & 1129.61514914326 & 100.05 & 1.30115375362026 \\
200.00 & 1129.54647220835 & 100.06 & 1.29876082766394 \\
200.00 & 1129.47779322423 & 100.07 & 1.29636990035784 \\
200.00 & 1129.40911219261 & 100.08 & 1.29398097228995 \\
200.00 & 1129.34042911522 & 100.09 & 1.29159404404834 \\
$\ldots$ & $\ldots$ & $\ldots$ & $\ldots$ \\
\hline
\end{tabular}




\begin{tabular}{cccc}
\hline 200.01 & 1057.49246048187 & 110.44 & 0.000108600045607765 \\
200.01 & 1057.42264487696 & 110.45 & $9.97254269521111 \mathrm{E}-05$ \\
200.01 & 1057.35282920624 & 110.46 & $9.34283808457733 \mathrm{E}-05$ \\
200.01 & 1057.28301347182 & 110.47 & $8.97094119600449 \mathrm{E}-05$ \\
200.01 & 1057.21319767581 & 110.48 & $8.85690248128939 \mathrm{E}-05$ \\
200.01 & 1057.14338182032 & 110.49 & $9.00077237762718 \mathrm{E}-05$ \\
200.01 & 1057.07356590748 & 110.50 & $9.40260130674855 \mathrm{E}-05$ \\
200.01 & 1057.00374993940 & 110.51 & 0.000100624396755625 \\
200.01 & 1056.93393391820 & 110.52 & 0.000109803378761529 \\
200.01 & 1056.86411784598 & 110.53 & 0.000121563462852541 \\
$\ldots$ & $\ldots$ & $\ldots$ & $\ldots$ \\
220.00 & 985.889620981968 & 119.91 & 0.867956024469972 \\
220.00 & 985.813424728890 & 119.92 & 0.870397390173534 \\
220.00 & 985.737230239742 & 119.93 & 0.872842322210716 \\
220.00 & 985.661037517254 & 119.94 & 0.875290821100765 \\
220.00 & 985.584846564157 & 119.95 & 0.877742887362299 \\
220.00 & 985.508657383183 & 119.96 & 0.880198521513648 \\
220.00 & 985.432469977063 & 119.97 & 0.882657724072978 \\
220.00 & 985.356284348531 & 119.98 & 0.885120495558091 \\
220.00 & 985.280100500320 & 119.99 & 0.887586836485938 \\
220.00 & 985.203918435163 & 120.00 & 0.890056747374229 \\
\hline & & &
\end{tabular}

5. The movement simulation of cross hinge four-bar mechanism

Taking the optimized dimension parameters of the cross hinge four-bar mechanism as the modeling dimension, the simulation model is established in Adams $/$ view $^{(14)(17)}$, displayed in Fig.8. Add rotation driving for the link 1 and the driving expression is inputted. The expression $15.0 d * \sin ($ time $* 360 d / 15)$, as shown in Fig.9, means the swing angle of the link 1 is $\pm 15^{\circ}$. Set terminate time for $15 \mathrm{~s}$, step for 100 , and conduct the movement simulation of the model.

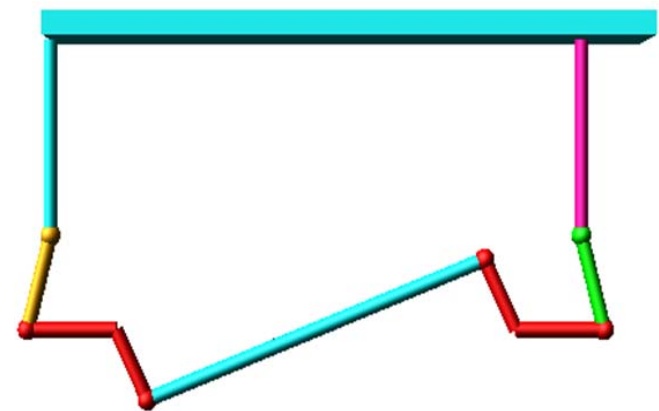

Fig. 8. The simulation model of cross hinge four-bar mechanism 


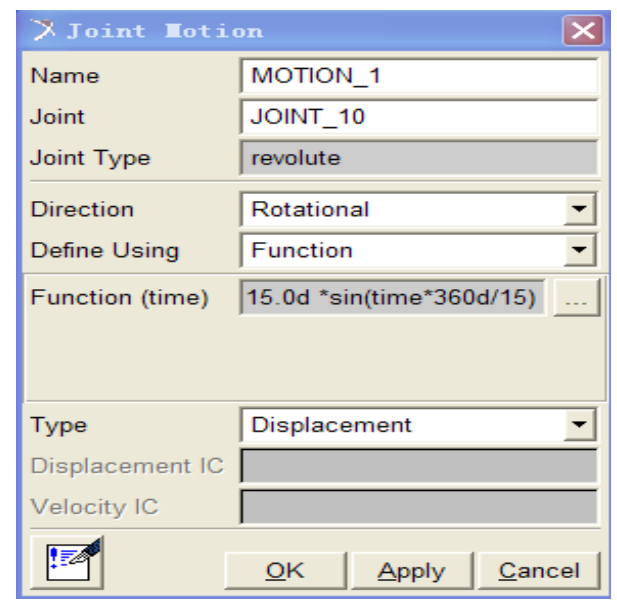

Fig.9. Add drive dialogue window

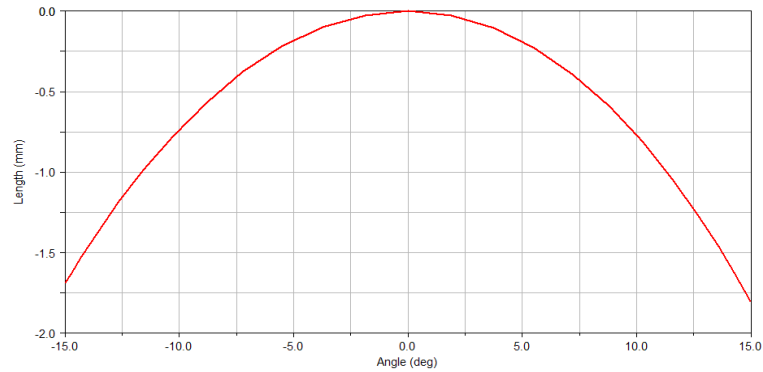

Fig.10. The displacement error curve between $\mathrm{E}$ and $\mathrm{F}$

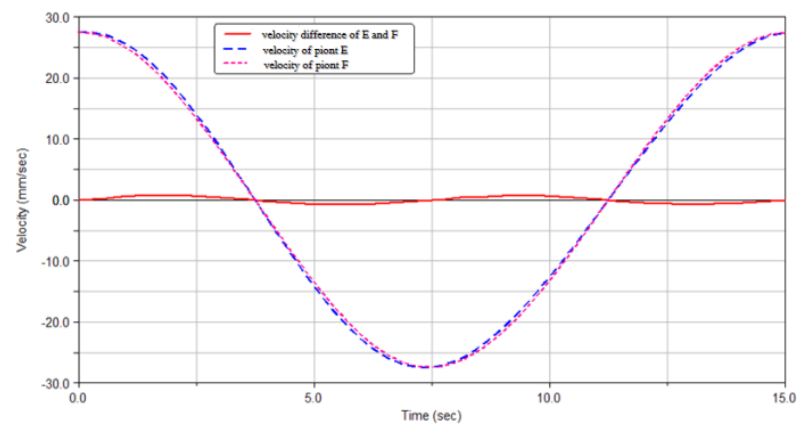

Fig.11. The speed and velocity error curve between $\mathrm{E}$ and $\mathrm{F}$

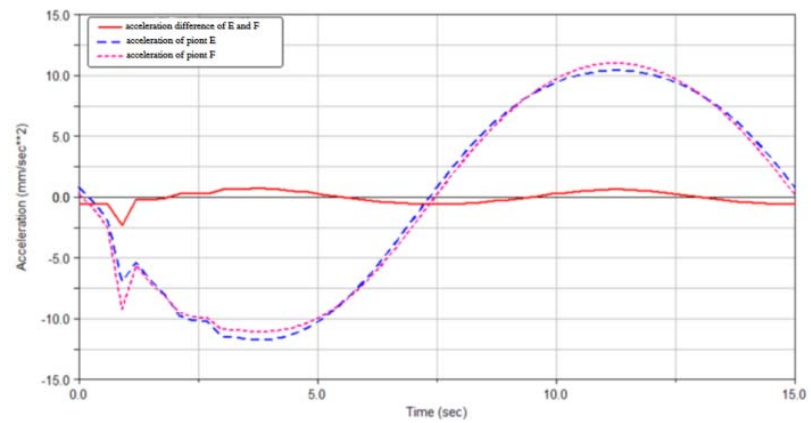

Fig.12. The acceleration and acceleration error curve between $\mathrm{E}$ and $\mathrm{F}$

Fig.10 indicates the displacement error curve between the points $\mathrm{E}$ and $\mathrm{F}$ in a wobble period. It can be determined from Fig. 10 that the error is approximately $1.6 \mathrm{~mm}$, and the length of the lifting platform GH is $1499.12 \mathrm{~mm}$. By calculating, the vertical movement margin of error of the lifting platform in a wobble period is approximately $0.06^{\circ}$. Figure 11 indicates the velocity curve of the points $\mathrm{E}$ and $\mathrm{F}$ and the velocity error curve between the two points in a wobble period. By calculating,, the error is approximately $2.76 \%$. Fig. 12 indicates the acceleration curve of the points $\mathrm{E}$ and $\mathrm{F}$ and the acceleration error curve between these two points in a wobble period. As shown in Fig.12, when $t=0.9 \mathrm{~s}$, the accelerations of points $\mathrm{A}$ and $\mathrm{B}$ change considerably. However, when $t \neq 0.9 s$, the 
changes in the accelerations of points $\mathrm{A}$ and $\mathrm{B}$ are not significant, the average error is approximately $6.11 \%$. These results indicate that a better optimization effect can be obtained by adopting the above optimization design method.

\section{Results}

(1) An optimization design method based on space conversion is proposed, which converts the nonlinear problem, based on different swing angles, into the linear problem based on the length change, reducing the difficulty of optimization design.

(2) When the relation model among length, angle and swing amplitude is established, through computer programming, the optimization design of the cross hinge four-bar mechanism is realized.

(3) The optimization design technology and computer aided technology are used so that the complex design work becomes more convenient and understandable. The optimal solution can be obtained quickly, and the design efficiency and quality are greatly improved.

(4) The Adams simulation is used to validate the effectiveness of the optimized data .

Currently this method has been applied in the processing equipment industry for footwear cutting, where better economic benefits have been realized.

\section{Acknowledgments}

The authors would like to thank Steven A. Blattner for his assistance in copy editing and proofreading the English translation of this article. The authors gratefully acknowledge the support by the "Six Talent Peak" Program of Jiangsu Province under Grant No.ZBZZ-043 and the National High-Tech. R\&D Program of China (863 Program) under Grants No. 2012AA110701.

\section{References}

(1) Michael E., Alfaro, Daniel I., et al. Evolutionary dynamics of complex biomechanical systems: an example of using the four-bar mechanism, Evolution, Vol.5, No.58(2007), pp.495-503.

(2) Tokuz Dulger L.C., Uyan S., Modelling, simulation and control of a four-bar mechanism with a brushless servo motor. Mechatronics, Vol.6, No.7(1997), pp.369-383.

(3) Karkoub M., Yigit A.S., Vibration control of a four-bar mechanism with a flexible coupler link. Journal of Sound and Vibration, Vol.4, No.222(1999), pp.171-189.

(4) LI Y.X., LIU Z., LI X.J., Computer aided modeling of planar linkage mechanism and development of general analytic software. Journal of Machine Design, Vol.23, No.5(2006), pp.58-60. (In Chinese)

(5) Chen X.B., Li Y.. Synthesis of CV Plane Hinge Linkages With Variable Distance Between the Input and Output Axies. Machine Design and Research, No.1(1998), pp. 31-32. (In Chinese)

(6) Sun X.B., Xiao R.B., Zhou J.. The Design Method and Its Application of Variable-Length-Four-BarLinkage. Journal of Huazhong University of Science and Technology, No.12(2000), pp.66-69. (In Chinese)

(7) Liu A.X., Yang T.L., Study on Optimum Shaking Force and Shaking Moment Balancing of Planar Linkages. Mechanical Science and Technology, Vol.16, No.4(1997), pp.647-652. (In Chinese)

(8) Liu G.G., Optimization Design of Four Bar Mechanism Based on the Improved Ant Colony Algorithm. Transactions of the Chinese Society for Agricultural Machinery, Vol. 37, 
No.1(2006), pp.149-151. (In Chinese)

(9) Weng G.R., Qian Z.L., Neural Network Synthesis of Four-bar Path Generating Linkages Based on Shape Spectrum. China Mechanical Engineering, Vol.16, No.3(2005), pp. 215-218. (In Chinese)

(10) SUN J.W., Ghu J.K., Synthesis of Coupler Curves of Spherical Four-bar Mechanism through Fast Fourier Transform. Chinese Journal of Mechanical Engineering, Vol.44, No.7(2008), pp.32-37. (In Chinese)

(11) Chu J.K., Sun J.W., Numerical atlas method for path generation of spherical four-bar mechanism. Mechanism and Machine Theory, Vol.45, No.6(2010), pp.867-879. (In Chinese)

(12) Radovan R. Bulatovića, Stevan R. Dordević. On the optimum synthesis of a four-bar linkage using differential evolution and method of variable controlled deviations. Mechanism and Machine Theory, Vol.44,No.1(2009), pp. 235-246.

(13) Chen X.B., Wang B., Chen M.F., et al. An innovative speed reduction mechanism with self-adaptability to variable transmission angles. Mechanism and Machine Theory, No.48(2012), pp.41-51.

(14) Yan S.J., Li F.G., Kinematics Simulation of Four Bar Linkage of Variable Bar Length. Journal of Mechanical Transmission, Vol. 35, No.12(2011), pp.46-48. (In Chinese)

(15) Khorshidi M., Soheilypour M., Peyro M., et al. Optimal design of four-bar mechanisms using a hybrid multi-objective GA with adaptive local search. Mechanism and Machine Theory, Vol. 46, No.10(2011), pp.1453-1465.

(16) Wang. B. Yancheng Institute of Technology. "The Optimization System of Cross Hinge Four-Bar Mechanism”, software copyright 2011R11L136304(2011).

(17) Pollayi H., Harursampath D., Geometrically non-linear dynamics of composite four-bar mechanisms. International Journal of Non-Linear Mechanics, Vol.47, No.8(2012), pp.837-850. 